\title{
Short-pulse length effects of the seed laser in high-gain harmonic generation free-electron laser
}

\author{
Deng haixiao (邓海啸), ${ }^{1,2}$ Wang xingtao (王兴涛), ${ }^{1}$ and Dai zhimin (戴志敏) ${ }^{1}$ \\ ${ }^{1}$ Shanghai Institute of Applied Physics, Chinese Academy of Sciences, Shanghai, 201800, People's Republic of China \\ ${ }^{2}$ Graduate University of the Chinese Academy of Sciences, Beijing, 100049, People's Republic of China
}

(Received 29 August 2007; published 29 April 2008)

\begin{abstract}
In order to generate coherent hard x-ray free-electron laser (FEL), cascading stages of high-gain harmonic generation (HGHG) scheme usually employ seed pulse with longitudinal length down to tens of femtoseconds. Such a short pulse length (SPL) seed laser, with broad bandwidth in the spectral domain, can no longer be characterized by monochromatic light in FEL equations. In this paper, a set of selfconsistent, multifrequency FEL equations is derived to describe the interaction between the seed laser and the electrons in the modulator of HGHG. Moreover, the SPL effects of the seed laser on the electron beam's energy modulation, the output pulse length, the output peak power, and the output wavelength tuning in HGHG are theoretically and numerically investigated. Study demonstrates that the SPL seed laser seriously and significantly influences the HGHG performances, and these influences have to be taken into account in the design and optimization of the seeded FEL scheme.
\end{abstract}

DOI: 10.1103/PhysRevSTAB.11.040703

PACS numbers: 41.60.Cr, 42.65.Ky

\section{INTRODUCTION}

Free-electron lasers (FELs) are devices that use the relativistic electron beams passing through a transverse periodic magnetic field in order to generate coherent electromagnetic radiation ranging from the infrared to the hard $\mathrm{x}$-ray regions. In recent years, taking self-amplified spontaneous emission (SASE) [1] and high-gain harmonic generation (HGHG) [2] as two leading candidates for approaching the deep ultraviolet to hard $\mathrm{x}$-ray spectral region, people are increasingly interested in the highgain, short-wavelength FEL. Benefited from the high quality seed laser, HGHG provides radiations with a high degree of stability whereas the central wavelength, bandwidth, and pulse duration can be controlled. These theoretical predictions have been demonstrated in the first HGHG proof of principle experiment [3].

According to the HGHG principle, to efficiently generate the coherent hard $\mathrm{x}$-ray radiation, the single-stage HGHG has to go to an ultrahigh harmonic number, which will make the gain length of the exponential growth region too large and reduce the stability. Cascading stages of HGHG scheme [4] with the "fresh bunch" technique [5] seem a feasible way to coherent hard $\mathrm{x}$-ray radiation. In a cascading scheme stating from the optical wavelength range, generally $\sim 5-6$ stages [4] of HGHG are needed to produce coherent radiation spanning to Angstrom wavelength range. Meanwhile, in order to obtain a high peak current at the entrance of the undulator system, the compress ratio of the electron bunch should be as high as $\sim 20-30$. Thus in hard $x$-ray FEL generated by the cascading HGHG technique, the practicable pulse length of the initial seed laser is usually in tens of femtoseconds order.

Early FEL theories and simulations $[2,6,7]$ are based on well-known monochromatic FEL equations. However, in HGHG FEL seeded by the short pulse length (SPL) laser, due to the broad bandwidth of the seed laser, the particle dynamics and the radiation propagation cannot be appropriately modeled by monochromatic FEL equations. In this paper, on the basis of multifrequency FEL equations, the SPL seed laser's effects in HGHG are theoretically estimated and numerically simulated. First in Sec. II, we briefly describe the physical processing of HGHG seeded by the SPL laser. In Sec. III, a set of self-consistent, multifrequency FEL equations is derived to properly picture the SPL seed laser's effects in HGHG and a new timedependent FEL code is developed. As a numerical example, the SPL seed laser's effects on $262 \mathrm{~nm}$ HGHG of the Shanghai deep ultraviolet (SDUV) FEL [8] are discussed in Sec. IV. Finally, we present our conclusions in Sec. V.

\section{THE SPL SEED LASER'S EFFECTS IN HGHG}

In HGHG, the electron beam's energy is modulated by the seed laser in the modulator and the energy modulation is converted into spatial modulation by the dispersive section, producing abundant harmonics bunching in the electron beam's density distribution. Then in the radiator which is designed to be resonant at the desired harmonic of the seed laser, rapid coherent emission at the resonant harmonic is produced. Further exponential amplification and ultimate saturation is achieved after reasonable radiator periods. Since the pulse duration of the SPL seed laser is much shorter than that of the electron beam, an electron bunch with a flattop beam current and infinite pulse duration is used in the following calculation.

\section{A. The electron beam's energy modulation}

Generally the modulator of HGHG works at the smallgain FEL interaction region, where the gain bandwidth is 


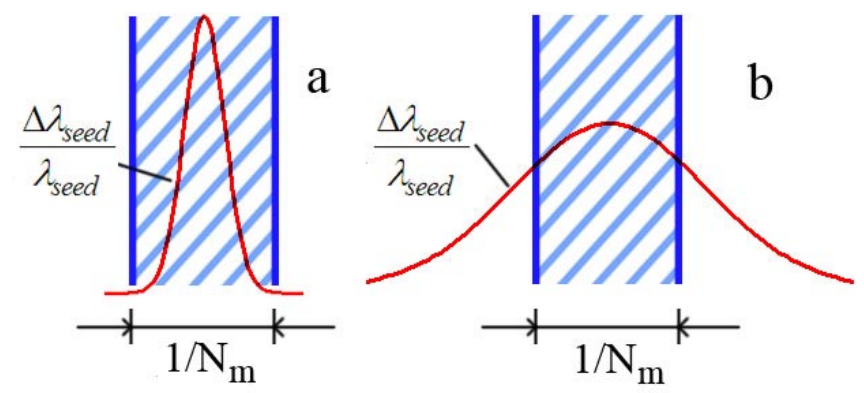

FIG. 1. (Color) The spectrum of the seed laser with respect to FEL gain bandwidth in the modulator.

characterized by the inverse of the undulator periods number. Accordingly, the gain bandwidth in the modulator is $1 / N_{m}$, where $N_{m}$ is the modulator periods number. It means that in the seed laser's spectrum, the parts which effectively contribute to the electron beam's energy modulation are the frequency parts that are below $1 / N_{m}$. Thus, if the seed laser's bandwidth $\Delta \lambda_{\text {seed }} / \lambda_{\text {seed }}$ is less than $1 / N_{m}$, as shown in Fig. 1(a), the seeding ability of the seed laser is fully exploited. This is the normal operation status of current HGHG FEL; on the other hand, when $\Delta \lambda_{\text {seed }} / \lambda_{\text {seed }}$ is much lager than $1 / N_{m}$, some parts in the seed laser's spectrum, out of the gain bandwidth, are useless to the electron beam's energy modulation, as depicted in Fig. 1(b). To describe the SPL effects of the seed laser on the electron beam's energy modulation, we evaluate the required SPL seed laser peak power $P_{\mathrm{SPL}}$ that leads to an equivalent bunching at the exit of the dispersive section in comparison with the peak power $P_{\text {seed }}$ predicted by the steady-state theory. A rough estimate is

$$
\frac{P_{\mathrm{SPL}}}{P_{\text {seed }}}=N_{m}\left(\frac{\Delta \lambda_{\text {seed }}}{\lambda_{\text {seed }}}-\frac{1}{N_{m}}\right) u\left(\frac{\Delta \lambda_{\text {seed }}}{\lambda_{\text {seed }}}-\frac{1}{N_{m}}\right)+1,
$$

where the step function $u(x)=1$ for $x \geq 0$ and $u(x)=0$ for $x<0$.

\section{B. The output wavelength tuning}

Output wavelength tuning is of great interest in HGHG FEL [9]. In most conventional HGHG FEL, the radiation from the laser system is split into two beams. One beam is utilized to drive a photocathode radiofrequency gun and the other is used as the seed laser for HGHG FEL. Such circumstances naturally allow accurate synchronization between the electron beam and the seed laser. However, the seed pulse length is usually several picoseconds and the output wavelength is determined by the central wavelength of the seed laser. Contrastively, the SPL seed laser with a bandwidth much broader than the FEL gain bandwidth may have a promising output wavelength tuning in HGHG. In Fig. 2, the red curve is the spectrum of the SPL seed laser and the blue shadow resembles the FEL gain bandwidth in the modulator. When we adjust the electron beam energy or the undulator gap, the resonant

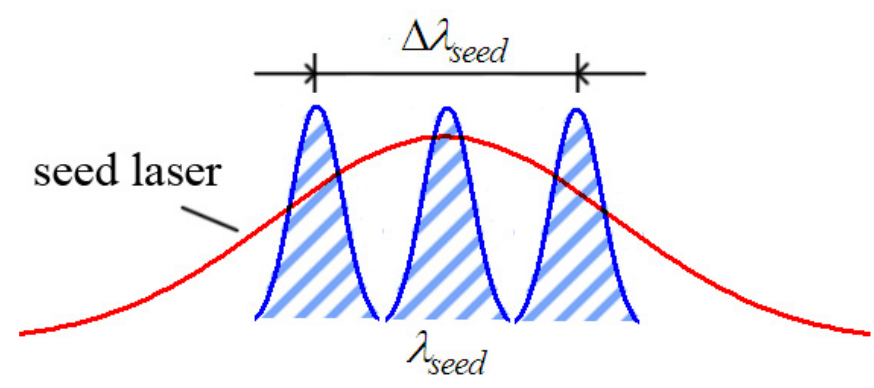

FIG. 2. (Color) Shift of the resonant wavelength in the modulator with respect to the spectrum of SPL seed laser.

wavelength in the modulator will shift. Then the electron beam will be bunched at a range of $\Delta \lambda_{\text {seed }}$ centered with $\lambda_{\text {seed }}$. The flexible electron bunching may be converted into the flexible output wavelength via a dispersion section and an alterable gap radiator. The tunability range $\Delta \lambda_{\text {out }} / \lambda_{\text {out }}$, including the effects of the SPL seed laser's bandwidth and the natural FEL bandwidth, may be roughly estimated as

$$
\left(\frac{\Delta \lambda_{\text {out }}}{\lambda_{\text {out }}}\right)^{2}=\left(\frac{\Delta \lambda_{\text {seed }}}{\lambda_{\text {seed }}}\right)^{2}+\left(\frac{1}{N_{r}}\right)^{2} \text {, }
$$

where $N_{r}$ is the undulator period number of the radiator. The second term can be neglected as it is much smaller than the first term in the case of the seed laser with pulse length down to tens of femtoseconds.

\section{The output pulse length}

According to the resonant condition, after traversing one period of the undulator, the radiation slips over the electron beam forward by the length of one radiation wavelength. This is called slippage effect. In the normal status of current HGHG FEL, as shown in Fig. 3(a), the seed pulse length is much longer than the slippage length in the modulator, thus the slippage effect usually can be neglected and the output pulse is expected to maintain the temporal properties of the seed pulse. However, HGHG seeded by the SPL laser operates close to the interaction regime where slippage effect becomes crucial. As illustrated in Fig. 3(b), after the modulator, the SPL seed laser only modulates the electrons which slip over it. Thus, the electron beam enters the radiator with a bunching pulse length that is basically equal to the slippage length $\tau_{M}$ in
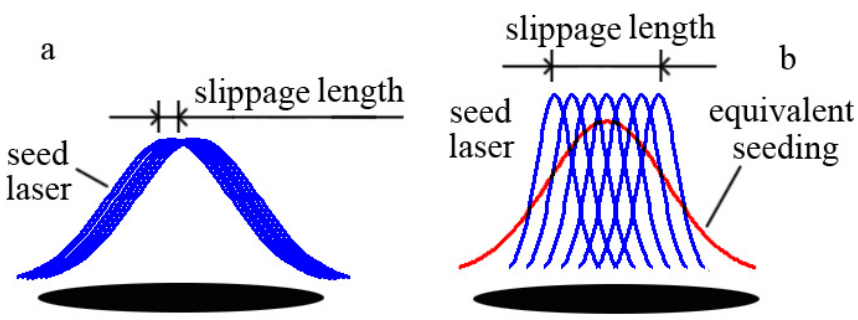

FIG. 3. (Color) The slippage length of the seed laser in the modulator with respect to the seed pulse length. 
the modulator, and the output pulse length $\Delta t_{\text {out }}$ in the radiator does not depend on the pulse length $\Delta t_{\text {seed }}$ of the SPL seed laser. Therefore, a rough estimate of the output pulse length can be given by

$$
\Delta t_{\text {out }}=\left(\Delta t_{\text {seed }}-\tau_{M}\right) u\left(\Delta t_{\text {seed }}-\tau_{M}\right)+\Delta t_{0}
$$

where $\Delta t_{0}$ is the constant pulse length caused by the slippage effect in the modulator. More accurately, $\Delta t_{\text {out }}$ is related to the slippage, superradiance, and other effects in the radiator, and its evolution in the radiator is complex. If the radiator length is properly chosen, in the radiator, the stretch of $\Delta t_{\text {out }}$ due to the slippage is usually counteracted by the reduction of $\Delta t_{\text {out }}$ due to the superradiance [10]. Thus, the radiator's effects on the output pulse length are neglected here.

\section{The output peak power}

The steady-state theory of FEL predicts that, after exponential growth, the radiation power saturates at a value that scales as $I^{4 / 3}[1]$, where $I$ is the peak current of the electron beam. However, in the radiator of HGHG seeded by the SPL laser, the pulse length of coherent radiation is much shorter than the slippage length $\tau_{R}$ in the radiator. As seen in Fig. 4, the peak of the coherent radiation quickly slips forward to the fresh part of the electron beam where the bunching is still small, and the steady-state condition is thereby destroyed. Thus, the power growth in the radiator, which relies on the integral of the electron beam bunching, will become slower. A radiation power growth with a rate less than predicted by the steady-state theory may be observed. The observed radiation power dependence upon the SPL seed laser can be modeled through an effective bunching "seen" by the radiation field as it slips through the electron bunch [11]. Thus for the Gaussian seed laser, the output peak power can be estimated as

$$
\frac{P_{\mathrm{out}}}{P}=\left(\frac{\int_{0}^{\tau_{R}} e^{-t^{2} / 2 /\left(\Delta t_{\mathrm{out}} / 2\right)^{2}} d t}{\tau_{R}}\right)^{4 / 3}
$$

where $P$ is the peak power of HGHG given by the steadystate theory, $P_{\text {out }}$ is the peak power of HGHG seeded by the SPL laser.
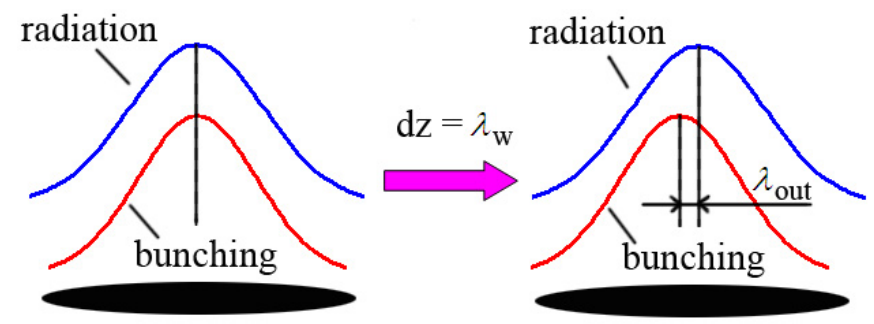

FIG. 4. (Color) Variation of the radiation pulse and the bunching pulse in the radiator.

\section{NUMERICAL APPROACHES OF SPL EFFECTS}

In current state-of-the-art FEL codes, polychromatic simulations operate purely in the domain and all frequency analysis is done afterwards using a postprocessor code. The slowly varying envelope approximation is utilized in the time and space for radiation propagation, but it cannot be used to appropriately describe the ultrashort laser pulse in the time domain; moreover, since a narrow bandwidth of frequencies around the central frequency is used to model the radiation, there exists difficulties in the validation of HGHG output wavelength tuning mentioned above. Thus, appropriate numerical approaches of HGHG FEL seeded by the SPL laser are discussed in this section.

\section{A. Description of SPL seed laser}

Conventionally, an ultrashort pulse laser is generated by a combination of different longitudinal modes, in which the frequency diversity $\Delta \omega$ and the phase diversity $\alpha$ of the adjacent mode are fixed. Suppose there are $2 N_{f}+1$ longitudinal modes with the same amplitude of $E_{0}$. We define that $q=0$ is the index of the central mode with an angular frequency of $\omega_{0}$ and an initial phase of 0 . Thus, we can write the $q$ th longitudinal mode as

$$
E_{q}(t)=E_{0} \cos \left[\left(\omega_{0}+q \Delta \omega\right) t+q \alpha\right]
$$

Usually $\alpha=0$ is chosen for simplicity, and the total field is the coherent addition of the $2 N_{f}+1$ modes

$$
E(t)=\sum_{q=-N_{f}}^{N_{f}} E_{q}(t)=A(t) \cos \left(\omega_{0} t\right),
$$

where $A(t)$ is the slow-varying amplitude, reads

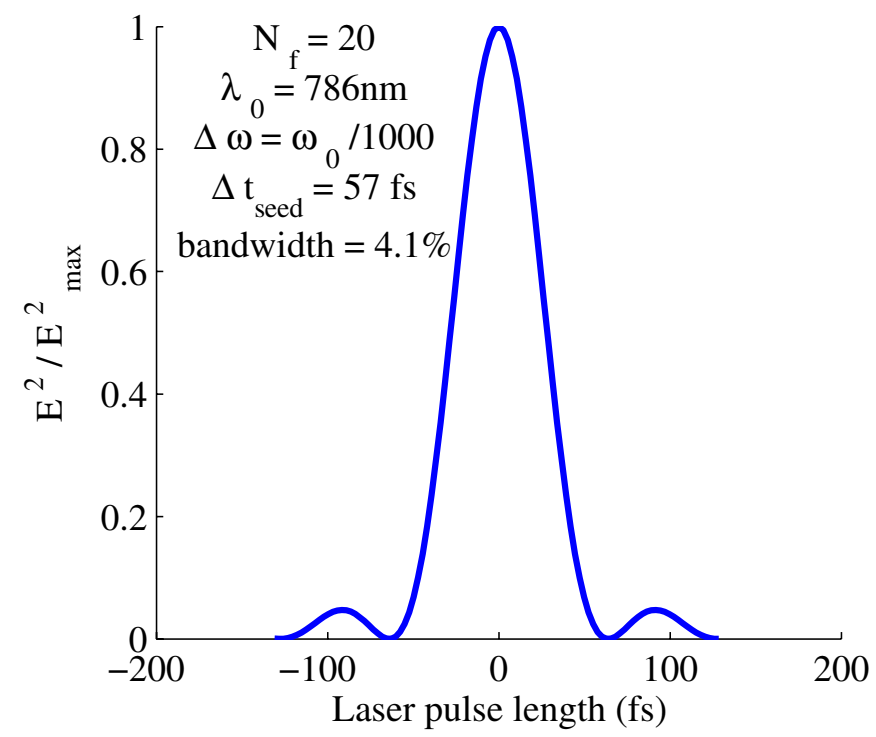

FIG. 5. (Color) SPL seed laser combined by 41 frequency modes, the central wavelength is $786 \mathrm{~nm}$. 


$$
A(t)=E_{0} \sin \left[\frac{1}{2}\left(2 N_{f}+1\right) \Delta \omega t\right] / \sin \left(\frac{1}{2} \Delta \omega t\right) .
$$

As illustrated in Fig. 5, the 786 nm laser pulse is combined by 41 longitudinal modes, where the 57 fs pulse length is constrained by the frequency spread in the spectrum via the causality principle. The temporal character of the SPL laser is characterized by $\Delta \omega / \omega_{0}$ and $N_{f}$ together, and the pulse length and the Fourier-transform-limited bandwidth of the SPL laser can be obtained by numerical calculations.

\section{B. Multifrequency FEL equations for HGHG}

Suppose a planar undulator with a sinusoidal magnetic field in the $y$ direction is given and considering a relativistic electron beam with the average energy $\gamma_{0} m c^{2}$ entering the modulator in the $z$ direction, one may observe the transverse wiggling motion, together with the "figure of eight" longitudinal phase oscillations in the resonant frame. The SPL seed laser is introduced as a linearly polarized electromagnetic field with multifrequency modes, as described above. We denote each angular frequency as $\omega_{q}$ and the slowly varying envelope function as $E_{q}(z)$. The corresponding wave number is $k_{q}=\omega_{q} / c$. Thus,

$$
E_{x}(z, t)=\frac{1}{2} \sum_{q=-N_{f}}^{N_{f}} E_{q}(z) e^{i\left(k_{q} z-\omega_{q} t\right)}+\text { c.c. }
$$

Normally there is no energy exchange between the electromagnetic field and the electron beam propagating in the same direction because their scalar product would be zero. Since there is a transverse component of the electron velocity in the undulator, there will be interaction between the electromagnetic field and the electron beam. The energy change is

$$
d W=m c^{2} d \gamma=e \vec{E}_{x} \cdot d \vec{r} .
$$

If the time derivative is taken away and the independent variable is changed from time to position,

$$
\frac{d \gamma_{j}}{d z}=-\frac{K}{2 \gamma m c^{2}}\left(\cos k_{w} z \sum_{q=-N_{f}}^{N_{f}} E_{q} e^{i\left(k_{q} z-\omega_{q} t\right)}+\text { c.c. }\right) \text {, }
$$

where $j$ runs over the electron, $K \equiv e A_{w} / m c$ is the undulator magnetic parameter, $\lambda_{w}$ is the period length of the undulator, and $k_{w}=2 \pi / \lambda_{w}$. Now we introduce $\theta=$ $k_{w} z+k_{r} z-\omega_{r} t+\xi \sin \left(2 k_{w} z\right)$ to describe the FEL bunching action, where $k_{r}$ is the resonant wave number in the modulator, $\omega_{r}=c k_{r}$ and $\xi=K^{2} /\left(4+2 K^{2}\right)$. The derivative of $\theta_{j}$ then becomes

$$
\frac{d \theta_{j}}{d z}=k_{w}\left(1-\frac{\gamma^{2}}{\gamma_{j}^{2}}\right) \cong 2 k_{w} \frac{\gamma_{j}-\gamma_{0}}{\gamma_{0}} .
$$

Then, the definition of $\theta$ is inserted into Eq. (10),

$$
\begin{aligned}
e^{i\left(k_{q} z-\omega_{q} t\right)} \cos k_{w} z= & \frac{1}{2}\left(e^{i\left(k_{w} z+k_{q} z-\omega_{q} t\right)}+e^{i\left(-k_{w} z+k_{q} z-\omega_{q} t\right)}\right) \\
= & \frac{1}{2} e^{i\left(\omega_{q} / \omega_{r}\right) \theta} e^{-i\left(\omega_{q} / \omega_{r}\right) \xi \sin 2 k_{w} z} \\
& \times\left(e^{i\left[1-\left(\omega_{q} / \omega_{r}\right)\right] k_{w} z}+e^{i\left[-1-\left(\omega_{q} / \omega_{r}\right)\right] k_{w} z}\right) .
\end{aligned}
$$

With the help of the expansion of the Bessel function,

$$
\begin{aligned}
e^{i\left(k_{q} z-\omega_{q} t\right)} \cos k_{w} z \cong & \frac{1}{2} e^{i\left(\omega_{q} / \omega_{r}\right) \theta} \sum_{p=-\infty}^{\infty} J_{P}\left[\frac{\omega_{q}}{\omega_{r}} \xi\right] \\
& \times e^{i\left(\left(\omega_{q} / \omega_{r}\right)-1\right) k_{w} z} \\
& \times\left(e^{-2 i p k_{w} z}+e^{-i(2+2 p) k_{w} z}\right) \\
= & \frac{1}{2} e^{i\left(\omega_{q} / \omega_{r}\right) \theta}[J J]_{q} .
\end{aligned}
$$

Now the energy exchange equation can be rewritten as

$$
\frac{d \gamma_{j}}{d z}=-\frac{K}{4 \gamma m c^{2}} \sum_{q=-N_{f}}^{N_{f}} E_{q} e^{i\left(\omega_{q} / \omega_{r}\right) \theta_{j}}[J J]_{q}+\text { c.c., }
$$

where $[J J]_{q}$ is the modified difference of the Bessel function, defined as

$$
[J J]_{q}=e^{i \pi\left[\left(\omega_{q} / \omega_{r}\right)-1\right]}\left(J_{0}\left[\xi \omega_{q} / \omega_{r}\right]-J_{1}\left[\xi \omega_{q} / \omega_{r}\right]\right) .
$$

For simplicity, the FEL universal scaling is introduced [12],

$$
\begin{gathered}
p_{j}=\left(\gamma_{j}-\gamma_{r}\right) / \rho \gamma_{r}, \quad A_{q}=\omega_{q} a_{q} / \omega_{p} \sqrt{\rho} \bar{\gamma}_{r}, \\
\bar{z}=2 \rho k_{w} z,
\end{gathered}
$$

where $\gamma_{r}=\left[\lambda_{w}\left(1+K^{2} / 2\right) / 2 \lambda_{r}\right]^{1 / 2}$ is the resonant energy, $\omega_{p}=\left(4 \pi e^{2} n_{e} / m\right)^{1 / 2}$ is the plasma frequency, $a_{q}=$ $e E_{q} \lambda_{q} / 2 \pi m c^{2}$ is the complex amplitude of the dimensionless radiation vector potential. The scaling of $A_{q}$ has been chosen so that $\left|A_{q}\right|^{2}=(1 / \rho)\left(\left|E_{q}\right|^{2} / 4 \pi n_{e} \gamma_{r} m c^{2}\right)$ is the FEL efficiency divided by $\rho, \rho=\left(a_{w} \omega_{p} / c k_{w}\right)^{2 / 3} / \gamma_{r}$ is the FEL parameter. Usually, the HGHG modulator works at the small-gain interaction region, and the slow-varying envelope $A_{q}$ can be approximated as a constant along the modulator. Thus, a set of equations to describe the FEL dynamics in the modulator is obtained, and can be expressed as

$$
\begin{gathered}
\frac{d \theta_{j}}{d \bar{z}}=p_{j} \quad \frac{d p_{j}}{d \bar{z}}=-\sum_{q=-N_{f}}^{N_{f}} A_{q} e^{i\left(\omega_{q} / \omega_{r}\right) \theta_{j}}[J J]_{q}+\text { c.c. }, \\
\frac{A}{A_{q}}=2 N_{f}+1 .
\end{gathered}
$$

After the modulator, when the energy-modulated electron beam enters the dispersive section, the dispersive section gives a rotation to the longitudinal phase space and changes the energy modulation to density modulation. The new coordinate $\left(p_{j}^{\prime}, \theta_{j}^{\prime}\right)$ is given by 


$$
p_{j}^{\prime}=p_{j} \quad \theta_{j}^{\prime}=n\left[\theta_{j}+\frac{\partial \theta}{\partial \gamma}\left(p_{j}-p_{0}\right)\right]
$$

where $n$ is frequency up-conversion number of HGHG and $\partial \theta / \partial \gamma$ is the strength of the dispersive section.

In comparison with the seed pulse length, as discussed in Sec. II, the output pulse length in the radiator will be much longer in HGHG seeded by the SPL laser. The radiation pulse in the radiator is about $\tau_{M} / \Delta t_{\text {seed }}$ times of the seed pulse length $\Delta t_{\text {seed }}$, hence the bandwidth in the radiator is $\Delta t_{\text {seed }} / n \tau_{M}$ of the seed bandwidth $\Delta \lambda_{\text {seed }} / \lambda_{\text {seed }}$. This indicates that the radiation bandwidth in the radiator is approximately $10 \%$ of the seed laser. Therefore the monochromatic FEL equation [1] is capable the FEL dynamics in the radiator.

\section{Development of FEL code for SPL effects}

A one-dimensional, time-dependent code on the basis of the multifrequency FEL equations derived above is built to numerically study the interactions between the electron beam and the SPL seed laser in the modulator. The seed laser pulse is represented by uniformly separated modes in the spectral domain, where we assume $\Delta \omega / \omega_{0}=1 / 1000$. The electron bunch is represented by uniform slices in the time domain, where the electron phase is loaded by "mirroring" method used in GENESIS [13] to remove the effect of finite electron numbers. The gain reduction due to the transverse emittance is equivalent to an external initial energy spread [14]. Moreover, to involve the slippage effect, a $-2 \pi$ offset is performed for each electron phase after traversing an undulator period. The FEL dynamics and radiation propagations in the radiator are solved by the conventional time-dependent simulation.

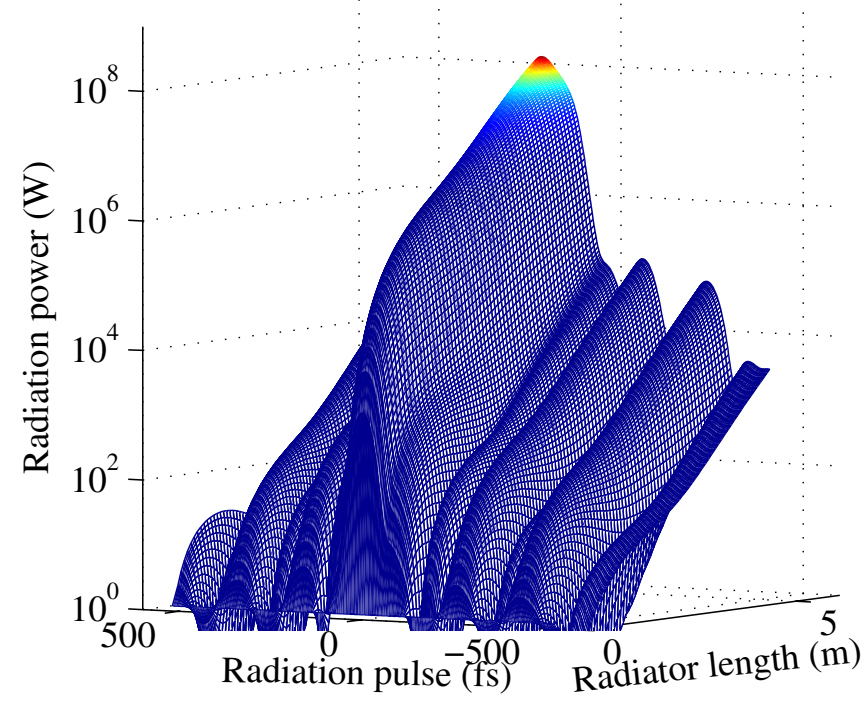

FIG. 6. (Color) The time-dependent output FEL pulse simulated by the newly developed FEL code.
To check the validity of such numerical approaches, the output pulse shape dependence on the seed laser is studied. As shown in Fig. 6, the simulated output pulse shape is very consistent with that of the seed laser. The origin of the quasi-sinc-type output pulse is the sinc seed signal shown in Fig. 5. Moreover, HGHG and SASE contributions can clearly be spotted in Fig. 6. In a further study of a Gaussian seed laser, we multiply the proper weighting factor to the dimensionless amplitude of each mode. The result is that all the secondary peaks of the output pulse disappear.

\section{SIMULATION PERFORMANCES OF SDUV FEL}

SDUV FEL is a $262 \mathrm{~nm}$ HGHG test facility. The design and relevant research and development have been under way since 2000. For the time being, the photocathode injector is under commissioning. It is designed to generate a $40 \mathrm{MeV}$ electron bunch with a charge of $1 \mathrm{nC}$ in $8 \mathrm{ps}$ length and a normalized emittance less than $6 \mathrm{~mm} \mathrm{mrad}$. This injector is expected to replace the existing $90 \mathrm{kV}$ nanosecond grid gun and the $15 \mathrm{MeV}$ buncher of the commissioned $100 \mathrm{MeV}$ Linac, and then the energy of the electron beam will be upgraded to $160 \mathrm{MeV}$. The fabrication of the bunch compressor, the radiator, the seed laser system, and the beam diagnostics have been completed. The main parameters of the scheme are listed in Table I. In this section, we numerically investigate the SPL seed laser's effects in SDUV FEL.

\section{A. The electron beam's energy modulation}

The pulse length of the $786 \mathrm{~nm}$ seed laser is $10 \mathrm{ps}$ in the normal operation status of SDUV FEL, which is long enough so that SPL effects can be neglected. According to the simulation results, $262 \mathrm{~nm}$ HGHG of SDUV FEL saturates at $5 \mathrm{~m}$ with a saturation power of $200 \mathrm{MW}$ when the seed laser power $P_{\text {seed }}=6.5 \mathrm{MW}$ and the dispersive

TABLE I. The main parameters of SDUV FEL.

\begin{tabular}{lc}
\hline \hline Modulator period length $\lambda_{w}$ & $50 \mathrm{~mm}$ \\
Modulator period number $N$ & 16 \\
Modulator undulator parameter $K$ & 2.04 \\
Modulator resonant wavelength $\lambda$ & $786 \mathrm{~nm}$ \\
Dispersive strength $\partial \theta / \partial \gamma$ & 1 \\
Radiator period length $\lambda_{w}$ & $25 \mathrm{~mm}$ \\
Radiator period number $N$ & 200 \\
Radiator undulator parameter $K$ & 1.45 \\
Radiator resonant wavelength $\lambda$ & $262 \mathrm{~nm}$ \\
Central wavelength of seed laser $\lambda_{s}$ & $786 \mathrm{~nm}$ \\
Peak power of seed laser $P_{\text {seed }}$ & $6.5 \mathrm{MW}$ \\
Beam energy $E$ & $160 \mathrm{MeV}$ \\
Beam peak current $I$ & $300 \mathrm{~A}$ \\
Beam transverse emittance $\varepsilon_{n}$ & $6 \mathrm{~mm} \mathrm{mrad}$ \\
Beam energy spread $\sigma_{\gamma} / \gamma$ & $0.01 \%$ \\
Average beta function & $3 \mathrm{~m}$ \\
Output wavelength $\lambda_{\text {out }}$ & $262 \mathrm{~nm}$ \\
\hline \hline
\end{tabular}




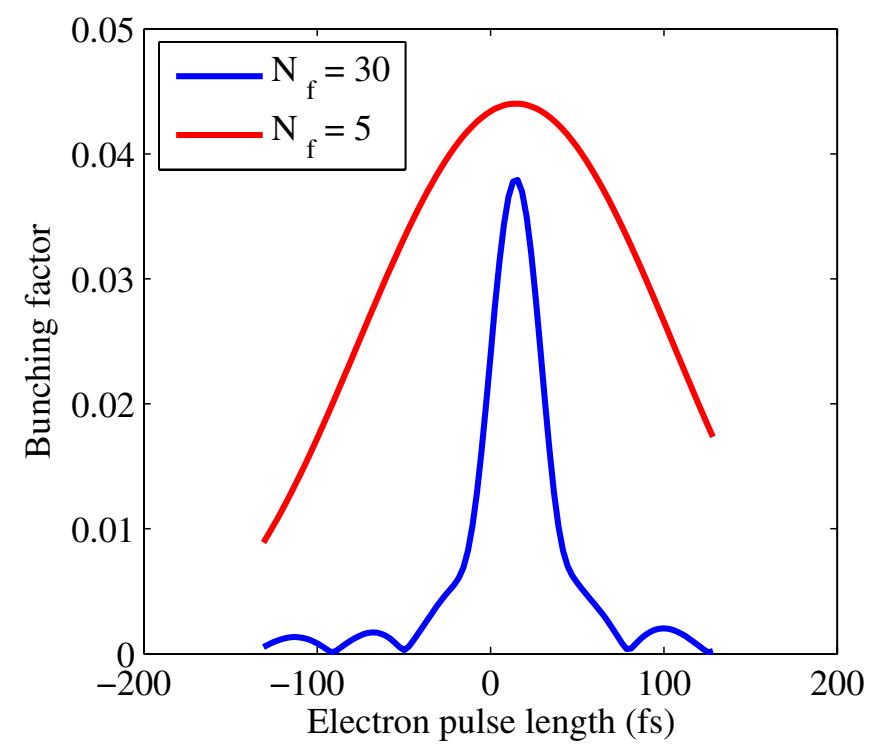

FIG. 7. (Color) Simulated bunching factor out of the dispersive section in different seed pulse length, with other parameters fixed.

strength $\partial \theta / \partial \gamma=1$. When the seed pulse length is decreased with the peak power fixed, the effective energy modulation in the modulator decreases, resulting in the reduction of the bunching factor after the dispersive section. In Fig. 7, the bunching factor after the dispersive section is illustrated for $\Delta t_{\text {seed }}=236$ and $38.5 \mathrm{fs}$, with $N_{f}=5$ and 30, respectively. In comparison with 0.46 in the steady-state case, the peak bunching factor reduces to 0.44 and 0.37 in the case of $\Delta t_{\text {seed }}=236$ and $38.5 \mathrm{fs}$, respectively.

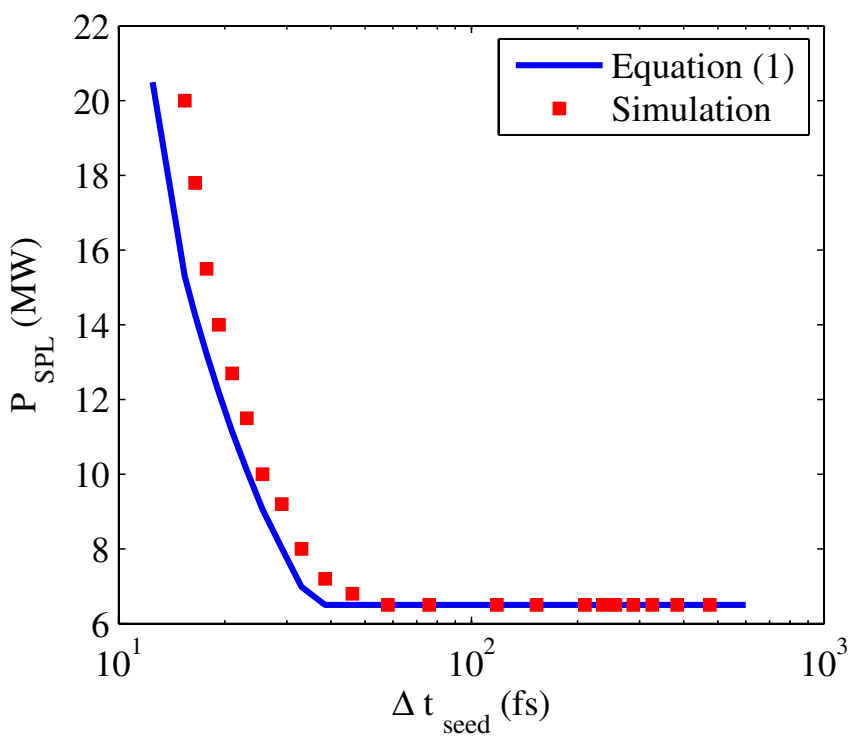

FIG. 8. (Color) The dependence of the required peak power of SDUV FEL on the seed pulse length, where the radiator length is $5 \mathrm{~m}$ and $\partial \theta / \partial \gamma=1$.
Thus, in order to obtain an equivalent peak bunching after the dispersive section, the peak power of the seed laser must be increased properly. As shown in Fig. 8, when $\Delta t_{\text {seed }}>45 \mathrm{fs}$, the required peak power $P_{\mathrm{SPL}}$ is almost constant, otherwise it would be reoptimized by Eq. (1). Further, we rewrite Eq. (1) after some brief calculation,

$$
\frac{P_{\mathrm{SPL}}}{P_{\text {seed }}}=\frac{1}{\Delta t_{\text {seed }}}\left(\tau_{M}-\Delta t_{\text {seed }}\right) u\left(\tau_{M}-\Delta t_{\text {seed }}\right)+1,
$$

where $P_{\mathrm{SPL}}$ is characterized by the peak power seen by the electron bunch due to slippage effects. Obviously, Eq. (15) is the temporal form of Eq. (1). Thus, the results in the time domain and in the spectral domain are consistent.

\section{B. The output wavelength tuning}

In SDUV FEL, a fixed gap radiator and an alterable gap modulator are employed. Therefore, if we adjust the electron beam energy to satisfy the resonant condition in the radiator and change the gap of the modulator to satisfy the resonant condition in the modulator, with the central wavelength of the SPL seed laser fixed, the output wavelength tuning is expected. In the simulation, we first numerically obtain the optimal seed laser power and dispersive strength for each wavelength. Then, on the basis of the optimal parameters, the output wavelength tuning is investigated. The simulated dependence of HGHG tunability range on the seed pulse length is shown in Fig. 9. The tunability range is basically in inverse proportion to the pulse length of the SPL seed laser, which is consistent with the theoretical estimates of Eq. (2). According to Fig. 9, a wavelength tunability range up to $15 \%$ can be attained by using a $15 \mathrm{fs}$ seed pulse with a central wavelength of $786 \mathrm{~nm}$.

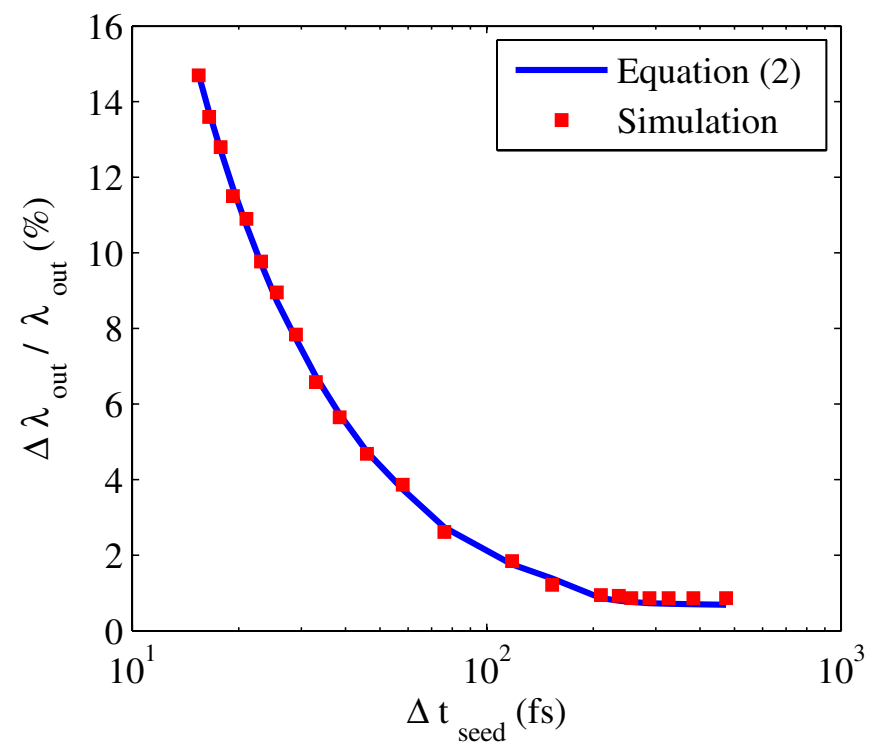

FIG. 9. (Color) The dependence of the tunability of SDUV FEL on the seed pulse length, where the radiator length is $5 \mathrm{~m}$ and $\partial \theta / \partial \gamma=1$. 
Since the continuous progress in the laser technology has made ultrashort, high intensity laser pulses available [15], by using the few-cycle laser, it may be possible to obtain a seeded FEL scheme with fully tunable output wavelength.

\section{The output pulse length}

The simulated output pulse length of SDUV FEL together with analytical estimates of Eq. (3) are depicted in Fig. 10. In the simulation, we use $P_{\mathrm{SPL}}$ as the power of the seed laser. When a long seed pulse is used, the output pulse length is approximately the pulse length of the seed laser. When the seed pulse length is less than the slippage length $\tau_{M}$ in the modulator, since strong slippage effects are involved, the output pulse length would be a constant value much lager than $\Delta t_{\text {seed }}$. In our case, the constant output pulse length $\Delta t_{0}$ is $65 \mathrm{fs}$, and the slippage length in the modulator is $41 \mathrm{fs}$. The difference between $\Delta t_{0}$ and $\tau_{M}$ is due to the complex evolution of the radiation pulse length in the radiator [10], which is not taken into account in Eq. (3).

\section{The output peak power}

Together with the analytical estimates of Eq. (4), the simulated dependence of the output peak power of $262 \mathrm{~nm}$ HGHG of SDUV FEL on the seed pulse length is given in Fig. 11. In the simulation, we use $P_{\text {SPL }}$ as the power of the seed laser. SDUV FEL saturates at $5 \mathrm{~m}$ in the radiator with a saturation power of $200 \mathrm{MW}$ when seeded by an ultralong laser, which is labeled by point A in Fig. 11. As the seed pulse length decreases, the steady-state dynamic between the radiation field and the electron beam is destroyed in the radiator. The gain reduction of the exponential

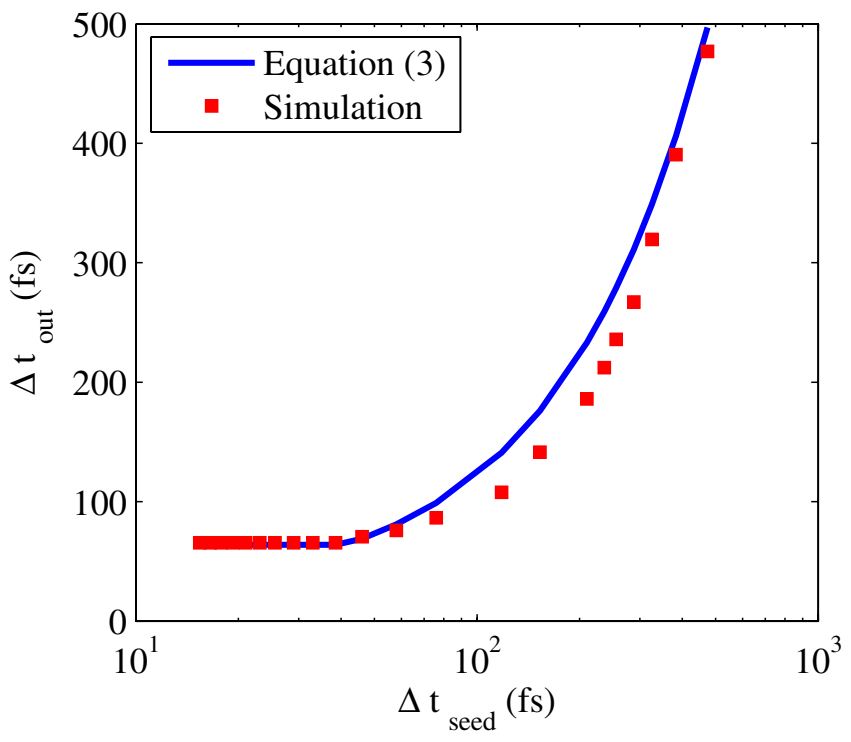

FIG. 10. (Color) The dependence of the output pulse length of SDUV FEL on the seed pulse length, where the radiator length is $5 \mathrm{~m}$ and $\partial \theta / \partial \gamma=1$.

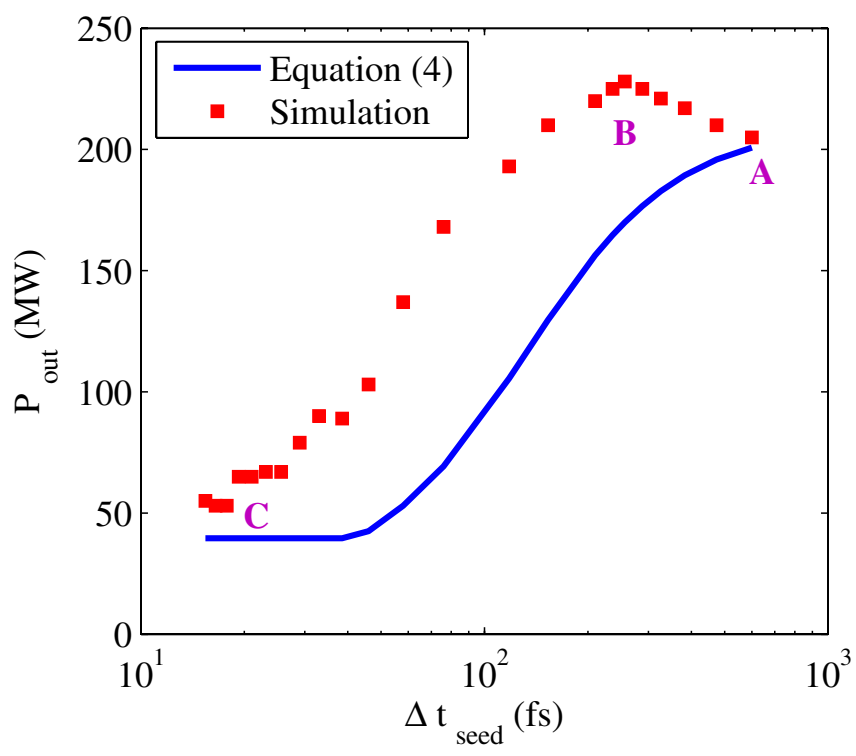

FIG. 11. (Color) The dependence of the output peak power of SDUV FEL on the seed pulse length, where the radiator length is $5 \mathrm{~m}$ and $\partial \theta / \partial \gamma=1$.

growth and the quadratic growth of superradiance come out together and compete with each other. Thus, with the seed pulse length decreasing, there exists a temporary and small increase of the output peak power, which can be explained by the quadratic growth of the superradiance that plays a more important role in power growth than FEL saturation in the steady-state theory. The exponential growth degradation becomes dominant from point $\mathrm{B}$, and the output peak power starts to decrease as predicted by Eq. (3), ultimately, when $\Delta t_{\text {seed }}<\tau_{M}$ at point $\mathrm{C}$, the output

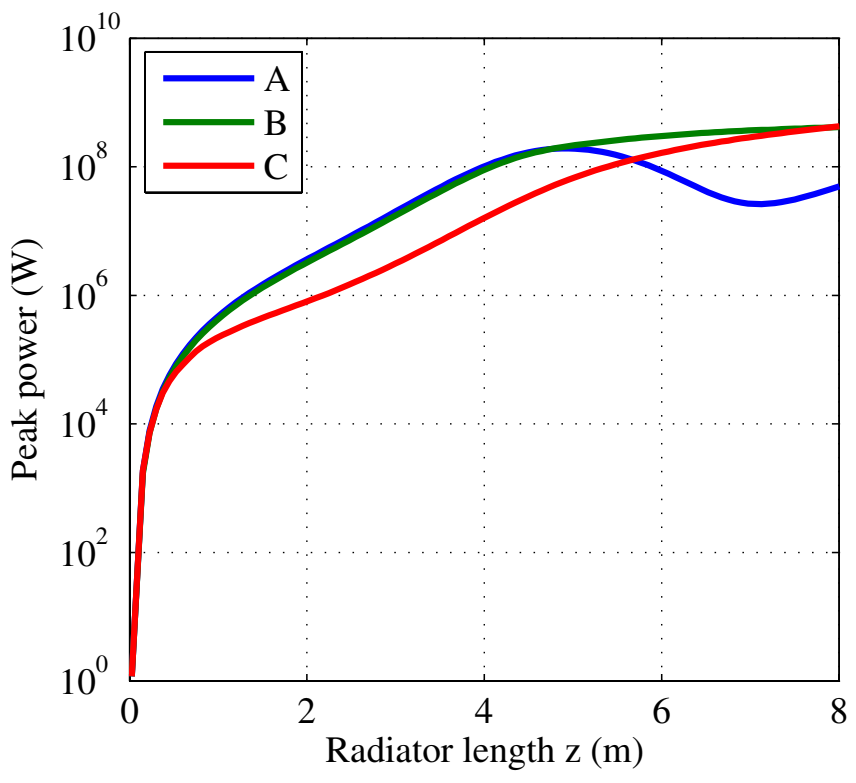

FIG. 12. (Color) The output peak power growth of the $262 \mathrm{~nm}$ HGHG of SDUV FEL in the radiator. 
pulse length is independent on $\Delta t_{\text {seed }}$, and the seed pulse length almost has almost no impact on the output peak power again. Compared to Fig. 11, an obvious discrepancy between the simulation results and the analytical estimate is observed. This is because the effects of superradiance have been neglected in Eq. (4), and the tiny increase caused by the superradiance does not appear in the analytical estimate. Therefore the simulated results agree well with the analytical estimates in the region where the exponential growth degradation dominates.

In order to clearly visualize what we have discussed above, we plot the output peak power growth in the radiator in Fig. 12, where A, B, and C represent the three points labeled in Fig. 11. Figure 12 strongly supports our statement. Further, one may observe that the quadratic growth of the superradiance still works until the growth of SASE stops the evolution of superradiance by ultimately spoiling the longitudinal phase space of the electrons.

\section{CONCLUDING REMARKS}

The SPL laser is typically obtained by the optical compression [15], resulting in an even broader bandwidth than theoretical estimate predicts. Once the SPL laser is employed as the seed of HGHG FEL, it may significantly influence HGHG performance. In this paper, theoretical analysis of the SPL seed laser's effects in single-stage HGHG are given. In order to validate the analytical estimates, a time-dependent FEL code on the basis of selfconsistent, multifrequency FEL equations is built to appropriately describe HGHG FEL seeded by the SPL laser. Using the new code, the SPL seed laser's effects on the electron beam's energy modulation, the output wavelength tuning, the output pulse length, and the output peak power are investigated. Our study shows a good agreement between the analytical estimates and numerical simulations.

On the way to generate coherent hard x-ray FEL, the SPL seed laser is usually employed in the cascading HGHG scheme with the "fresh bunch" technique. The SPL seed laser's effects are important in cascading
HGHG FEL. Some more detailed work on this issue needs to be carried out and a dedicated discussion will be given in the future.

\section{ACKNOWLEDGMENTS}

The authors are grateful to $\mathrm{H}$. Bei and D. Wang for useful discussions. This work is partially supported by the Major State Basic Research Development Program of China under Grant No. 2002CB713600, the Chinese Academy of Sciences, and the National Natural Science Foundation of China.

[1] J. Murphy, C. Pellegrini, and R. Bonifacio, Opt. Commun. 53, 197 (1985).

[2] L. H. Yu, Phys. Rev. A 44, 5178 (1991).

[3] L. H. Yu et al., Nucl. Instrum. Methods Phys. Res., Sect. A 445, 301 (2000).

[4] J. H. Wu and L. H. Yu, Nucl. Instrum. Methods Phys. Res., Sect. A 475, 104 (2001).

[5] I. Ben-Zvi et al., Nucl. Instrum. Methods Phys. Res., Sect. A 318, 726 (1992).

[6] L. H. Yu and J. H. Wu, Nucl. Instrum. Methods Phys. Res., Sect. A 483, 493 (2002).

[7] L. H. Yu and J. H. Wu, Nucl. Instrum. Methods Phys. Res., Sect. A 507, 455 (2003).

[8] Z. T. Zhao et al., Nucl. Instrum. Methods Phys. Res., Sect. A 528, 591 (2004).

[9] T. Shaftan and L. H. Yu, Phys. Rev. E 71, 046501 (2005).

[10] L. Giannessi and P. Musumeci, New J. Phys. 8, 294 (2006).

[11] Z. Huang and W. M. Fawley, in IEEE Particle Accelerator Conference (IEEE, Chicago, IL, 2001), pp. 2713-2715.

[12] R. Bonifacio, L. M. Narducci, and C. Pellegrini, Opt. Commun. 50, 373 (1984).

[13] S. Reiche, Nucl. Instrum. Methods Phys. Res., Sect. A 429, 243 (1999).

[14] T. M. Tran and J. S. Wurtele, Comput. Phys. Commun. 54, 263 (1989).

[15] T. Brabec and F. Krausz, Rev. Mod. Phys. 72, 545 (2000). 